\title{
Os impactos da pandemia de COVID-19 na mobilidade urbana: uma revisão
}

\section{narrativa de literatura}

\author{
The impacts of the COVID-19 pandemic on urban mobility: a narrative literature review \\ Los impactos de la pandemia de COVID-19 en la movilidad urbana: una revisión narrativa de la
}

literatura

Recebido: 25/11/2021 | Revisado: 02/12/2021 | Aceito: 07/12/2021 | Publicado: 16/12/2021

Francisca Rafaela Pereira de Amorim Castro Rosa

ORCID: https://orcid.org/0000-0002-6775-0848 Instituto Master de Ensino Presidente Antônio Carlos, Brasil E-mail: rafaela23_amorim@hotmail.com Bárbara Queiroz de Figueiredo ORCID: https://orcid.org/0000-0003-1630-4597 Centro Universitário de Patos de Minas, Brasil

E-mail: barbarafigueiredo@unipam.edu.br

Bruno Rodrigues Cancela

ORCID: https://orcid.org/0000-0002-1348-1398

Universidade Federal do Rio de Janeiro, Brasil

E-mail: brcancela@gmail.com

Dieison Danrlei Roehrs

ORCID: https://orcid.org/0000-0003-2919-9765

Centro Universitário de Goiatuba, Brasil E-mail: dieisondanrlei1 @ hotmail.vom

João Victor Marques de Matos

ORCID: https://orcid.org/0000-0002-4463-3462

Centro Universitário de Goiatuba, Brasil

E-mail: joaovictormtos@hotmail.com

Luanna Oliveira Gonçalves

ORCID: https://orcid.org/0000-0002-4463-3462 Instituto Master de Ensino Presidente Antônio Carlos, Brasil E-mail: luanna435@gmail.com

Vinícius Leandro Oliveira Medeiros

ORCID: https://orcid.org/0000-0003-1422-7452

Universidade Católica de Brasília, Brasil

E-mail: vinyleandro.bsb@outlook.com

\begin{abstract}
Resumo
Introdução: a mobilidade urbana é entendida como a capacidade cotidiana de deslocamento de pessoas e bens no espaço urbano de forma segura e em tempo considerado adequado. Recentemente, a situação pandêmica foi gradualmente amenizada e o plano de recuperação econômica e social foi colocado em pauta pelo governo durante o período pós-epidemia. Nessas circunstâncias, o sistema de transporte urbano desempenha um papel crucial no processo de recuperação social e econômica como garantia básica da cidade. Portanto, maior atenção deve ser dada aos impactos da pandemia de COVID-19 no sistema de transporte urbano, bem como no comportamento de viagem. Objetivo: evidenciar os impactos causados pela pandemia de COVID-19 na mobilidade urbana. Metodologia: trata-se de revisão narrativa da literatura, com estudos acoplados nas seguintes bases de dados: National Library of Medicine (PubMed MEDLINE), Scientific Electronic Library Online (Scielo), Cochrane Database of Systematic Reviews (CDSR), Google Scholar, Biblioteca Virtual em Saúde (BVS) e EBSCO Information Services. Resultados: alguns impactos sobre a mobilidade urbana puderam ser observados devido ao alto grau de contágio do novo coronavírus e, portanto, à necessidade de permanecer em casa e manter um distanciamento mínimo. São impactos a princípio sociais, econômicos ou ambientais, mas que apresentam forte relação com a mobilidade urbana. Em geral houve uma drástica diminuição da locomoção nas cidades, que se encontraram vazias, e há um temor com relação à volta às atividades normais à medida que o isolamento vai sendo flexibilizado. Considerações finais: a pandemia de COVID-19 provocou inúmeras mudanças na vida da população mundial. Na mobilidade urbana não foi diferente. Com o fechamento do comércio considerado não essencial, a suspensão de aulas e a instalação de regimes de teletrabalho, grande parte dos deslocamentos diários foram interrompidos e/ou modificados. Medidas como a instalação de políticas de subsídio do transporte público, de ampliação das ciclovias, de aplicação de ações de desestímulo do uso do transporte individual motorizado, de melhora na segurança pública e de circulação de pedestres e ciclistas etc., se mostraram urgentes durante a quarentena, não apenas para gerar condições mínimas para mobilidade ativa, mas também para melhorar a qualidade de vida da população como um todo.
\end{abstract}

Palavras-chave: COVID-19; Pandemia; Mobilidade urbana; Deslocamento; Impactos. 


\begin{abstract}
Introduction: urban mobility is understood as the daily ability to move people and goods in the urban space safely and in a time considered appropriate. Recently, the pandemic situation was gradually eased and the economic and social recovery plan was put on the agenda by the government during the post-epidemic period. In these circumstances, the urban transport system plays a crucial role in the process of social and economic recovery as a basic guarantee of the city. Therefore, greater attention must be paid to the impacts of the COVID-19 pandemic on the urban transport system, as well as on travel behavior. Objective: to highlight the impacts caused by the COVID-19 pandemic on urban mobility. Methodology: this is a narrative review of the literature, with studies linked to the following databases: National Library of Medicine (PubMed MEDLINE), Scientific Electronic Library Online (Scielo), Cochrane Database of Systematic Reviews (CDSR), Google Scholar, Library Health Virtual (BVS) and EBSCO Information Services. Results: some impacts on urban mobility could be observed due to the high degree of contagion of the new coronavirus and, therefore, the need to stay at home and keep a minimum distance. These are initially social, economic or environmental impacts, but they have a strong relationship with urban mobility. In general, there has been a drastic decrease in locomotion in cities, which have found themselves empty, and there is fear of returning to normal activities as isolation is eased. Final considerations: the COVID-19 pandemic caused countless changes in the lives of the world population. In urban mobility it was no different. With the closing of businesses considered nonessential, the suspension of classes and the installation of teleworking systems, a large part of daily commuting was interrupted and/or modified. Measures such as the installation of public transport subsidy policies, the expansion of bicycle lanes, the application of actions to discourage the use of individual motorized transport, improvement in public safety and the circulation of pedestrians and cyclists, etc., proved to be urgent during the quarantine, not only to create minimal conditions for active mobility, but also to improve the quality of life of the population as a whole.
\end{abstract}

Keywords: COVID-19; Pandemic; Urban mobility; Displacement; Impacts.

\title{
Resumen
}

Introducción: la movilidad urbana se entiende como la capacidad diaria de trasladar personas y mercancías en el espacio urbano de forma segura y en un tiempo que se considere oportuno. Recientemente, la situación de la pandemia se alivió gradualmente y el gobierno puso en la agenda el plan de recuperación económica y social durante el período posterior a la epidemia. En estas circunstancias, el sistema de transporte urbano juega un papel crucial en el proceso de recuperación social y económica como garantía básica de la ciudad. Por lo tanto, se debe prestar mayor atención a los impactos de la pandemia COVID-19 en el sistema de transporte urbano, así como en el comportamiento de los viajes. Objetivo: destacar los impactos provocados por la pandemia COVID-19 en la movilidad urbana. Metodología: se trata de una revisión narrativa de la literatura, con estudios vinculados a las siguientes bases de datos: National Library of Medicine (PubMed MEDLINE), Scientific Electronic Library Online (Scielo), Cochrane Database of Systematic Reviews (CDSR), Google Scholar, Library Health Servicios de información virtual (BVS) y EBSCO. Resultados: se pudieron observar algunos impactos en la movilidad urbana por el alto grado de contagio del nuevo coronavirus y, por tanto, la necesidad de permanecer en casa y mantener una distancia mínima. Se trata inicialmente de impactos sociales, económicos o ambientales, pero tienen una fuerte relación con la movilidad urbana. En general, ha habido una disminución drástica de la locomoción en las ciudades, que se han encontrado vacías, y existe el temor de volver a las actividades normales a medida que se alivia el aislamiento. Consideraciones finales: la pandemia de COVID-19 provocó innumerables cambios en la vida de la población mundial. En movilidad urbana no fue diferente. Con el cierre de negocios considerados no imprescindibles, la suspensión de clases y la instalación de sistemas de teletrabajo, se interrumpió y / o modificó gran parte de los desplazamientos diarios. Medidas como la implantación de políticas de subvenciones al transporte público, la ampliación de ciclovías, la aplicación de acciones para desalentar el uso del transporte motorizado individual, la mejora de la seguridad ciudadana y la circulación de peatones y ciclistas, etc., resultaron urgentes durante la cuarentena, no solo para crear las condiciones mínimas para la movilidad activa, sino también para mejorar la calidad de vida de la población en su conjunto.

Palabras clave: COVID-19; Pandemia; Movilidad urbana; Desplazamiento; Impactos.

\section{Introdução}

A mobilidade urbana é entendida como a capacidade cotidiana de deslocamento de pessoas e bens no espaço urbano de forma segura e em tempo considerado adequado. Nos últimos anos, essa questão tem se mostrado extremamente problemática na maioria das cidades, ocasionada pelo crescimento acelerado e desordenado, impedindo planejamento e estruturação adequados e, consequentemente, comprometendo a mobilidade e a saúde da população. Entende-se que esta questão influência fortemente o processo saúde-doença, ainda que muitas das causas e ações que poderiam ser realizadas para melhorar a situação não possam sofrer interferência direta da equipe de saúde, podendo limitar sua capacidade de ação caso não ocorra uma articulação efetiva com outros setores envolvidos. Em alguns municípios brasileiros foram identificados 
problemas como fluxo intenso de veículos, falta de sinalização, ruas sem acostamento, falta de calçadas e ciclovias, etc., como situações de risco para a comunidade (Huang et al., 2020).

A pandemia abrupta de COVID-19 interrompeu o desenvolvimento econômico e social normal na China e em todo o mundo, especialmente para as megacidades por causa de suas grandes populações. Medidas rígidas de controle da epidemia têm sido adotadas em muitas cidades, como bloqueio e suspensão do trabalho devido à necessidade de prevenção e controle da epidemia, que têm desempenhado um papel importante no controle da disseminação rápida e em larga escala da COVID-19. Sem dúvida, essas políticas de controle da epidemia reduziram o número de atividades sociais e econômicas e tiveram impactos significativos nos sistemas de transporte urbano, como a diminuição repentina de viagens e a mudança de modo de transporte. Recentemente, a situação pandêmica foi gradualmente amenizada e o plano de recuperação econômica e social foi colocado em pauta pelo governo durante o período pós-epidemia. Nessas circunstâncias, o sistema de transporte urbano desempenha um papel crucial no processo de recuperação social e econômica como garantia básica da cidade. Portanto, maior atenção deve ser dada aos impactos da pandemia de COVID-19 no sistema de transporte urbano, bem como no comportamento de viagem (Laverty et al., 2020). Aliado a isso, nota-se que a avaliação do impacto da pandemia nos transportes públicos e na economia social é muito importante para o trabalho de reconstrução no período pós-epidemia, e o objetivo deste estudo foi de evidenciar os impactos causados pela pandemia de COVID-19 na mobilidade urbana.

\section{Metodologia}

Trata-se de uma pesquisa descritiva do tipo revisão narrativa da literatura, que buscou evidenciar os impactos causados pela pandemia de COVID-19 na mobilidade urbana. A pesquisa foi realizada através do acesso online nas bases de dados National Library of Medicine (PubMed MEDLINE), Scientific Electronic Library Online (Scielo), Cochrane Database of Systematic Reviews (CDSR), Google Scholar, Biblioteca Virtual em Saúde (BVS) e EBSCO Information Services, no mês de novembro de 2021. Para a busca das obras foram utilizadas as palavras-chaves presentes nos descritores em Ciências da Saúde (DeCS): em português: "mobilidade urbana", "COVID-19", "transporte", "impactos" e em inglês: "urban mobility", "COVID-19", "transport", "impacts". Como critérios de inclusão, foram considerados artigos originais, que abordassem o tema pesquisado e permitissem acesso integral ao conteúdo do estudo, publicados no período de 2020 a 2021 , em inglês e português. O critério de exclusão foi imposto naqueles trabalhos que não estavam em inglês e espanhol, que não tinham passado por processo de Peer-View e que não abordassem o tema. A estratégia de seleção dos artigos seguiu as seguintes etapas: busca nas bases de dados selecionadas; leitura dos títulos de todos os artigos encontrados e exclusão daqueles que não abordavam o assunto; leitura crítica dos resumos dos artigos e leitura na íntegra dos artigos selecionados nas etapas anteriores. Assim, totalizaram-se 11 artigos científicos para a revisão narrativa da literatura, com os descritores apresentados acima, dos últimos doze anos e em língua portuguesa.

\section{Resultados e discussão}

Alguns impactos sobre a mobilidade urbana puderam ser observados devido ao alto grau de contágio do SARS-CoV-2 e, portanto, à necessidade de permanecer em casa e manter um distanciamento mínimo. São impactos a princípio sociais, econômicos ou ambientais, mas que apresentam forte relação com a mobilidade urbana. Em geral, houve uma drástica diminuição da locomoção nas cidades, que se encontraram vazias, bem como houve um temor com relação à volta às atividades normais à medida que o isolamento vai sendo flexibilizado (Hadjidemetriou et al., 2021).

A COVID-19 costuma demandar um atendimento médico incomum: em vez de serem territorializados (como acontece fora dos tempos de pandemia), os locais de referência são centralizados, seja para evitar a disseminação do vírus por diversos espaços, seja porque em alguns casos há necessidade de intervenção intensiva, para controle dos sinais vitais e auxílio 
de respiradores, por exemplo. Assim, pacientes vulneráveis, que em geral têm atenção de saúde próxima de seu local de moradia, se veem diante de uma barreira logística e geográfica no acesso ao atendimento médico. Quando analisa-se a problemática da mobilidade urbana, observa-se o mesmo quadro de assimetria e desigualdade social encontrada em outras áreas - renda, habitação, saneamento, coleta de lixo, e de longa data sabemos que a mobilidade urbana cresce diretamente proporcional à renda das pessoas (Chinazi et al., 2020).

A demanda pelo transporte público caiu drasticamente com o início do isolamento social, seja pelo medo da doença ou por regulamentação governamental para que as pessoas ficassem em casa. Mesmo com a flexibilização do isolamento, a demanda por esse tipo de transporte é consideravelmente menor do que antes da pandemia, pois contribui para a formação de aglomerações ao mesmo tempo em que há a necessidade de manter um distanciamento social mínimo enquanto não houver vacina ou cura para a COVID-19. Com isso, a receita das operadoras de transporte público cai, podendo comprometer o serviço, por falência das empresas ou aumento das tarifas, por exemplo. Nesse momento, é nítida a necessidade de subsídios para o transporte público (Kraemer et al., 2020).

Neste cenário, há em geral uma preferência pelo transporte individual motorizado ao transporte público. No Brasil, segundo Ximenes et al. (2020), 58\% dos brasileiros entrevistados pretendem se deslocar pela cidade por meio de carro particular após a pandemia. A pesquisa afirma que essa escolha pode retratar também a realidade de grandes trajetos no dia a dia, que demonstram baixa heterogeneidade e má distribuição dos serviços urbanos. Se outra alternativa não for oferecida, a opção pelo carro certamente vai piorar consideravelmente os problemas de congestionamentos e de poluição atmosférica nas cidades. Outrossim, por ser um meio de transporte de baixo custo e seguro, do ponto de vista do distanciamento entre as pessoas e por ser individual, a bicicleta vem sendo uma alternativa muito adotada pelas pessoas ao redor do mundo desde que a pandemia começou, e, por isso a importância de se investir em ciclovias, por exemplo (Aloi et al., 2020).

Os deslocamentos das pessoas de baixa renda estão diretamente associados ao trabalho que, quando formalizado, garante a elas o acesso ao Vale Transporte, por exemplo. Aliado a isso, os trabalhadores informais carecem deste apoio e a crise econômica que vive-se desde 2020, que já colocou milhares de pessoas fora de empregos formais. Outrossim, o crescimento do número de moradores de rua também vem ocorrendo devido aos custos elevados do transporte no orçamento da família e da instabilidade da renda daqueles que trabalham sem vínculo formal de emprego. Outro aspecto a se destacar é a importância da presença das mulheres nestas comunidades, em que muitas delas respondem por uma parcela significativa ou mesmo total da renda das famílias, dos cuidados das crianças e dos idosos, e embora as diferenças salariais entre gêneros ainda sejam significativas, a formalização do trabalho doméstico e das cuidadoras de idosos, trouxe muitas delas para o transporte público principalmente pelo acesso ao Vale Transporte, processo que pode ser interrompido (Huang et al., 2020).

Ademais, os segmentos da população de baixa renda já vivem em regime de isolamento, quando refere-se à mobilidade urbana. As viagens de lazer nos finais de semana requerem um esforço suplementar, que pode significar a falta de recursos no final do mês, por exemplo. Por isso, iniciativas de tarifa zero nos domingos tem sido exitosos em muitos municípios, pois permitem com que as famílias se desloquem para visitar parentes, parques e outros atrativos de qual são carentes a maior parte dos bairros populares. Ou seja, o isolamento ou distanciamento social precisa ser considerado a partir da realidade de cada segmento da sociedade brasileira, o que requer ações direcionadas a cada um deles, de modo a tornar factíveis a adoção das medidas necessárias para a proteção das pessoas da contaminação pelo COVID-19. Além disso, a necessidade de permanecer em casa obrigou muitas pessoas a trabalharem em sistema de home office, que foi um desafio para alguns, mas que vem sendo uma alternativa vantajosa para muitas pessoas e empresas (Arellana et al., 2020).

Do ponto de vista das populações que vivem em comunidades e favelas, muitas medidas adotadas pelas autoridades de transporte público e empresas operadoras já estão sendo implementadas. A primeira delas foi a defesa da manutenção dos serviços de transporte público ajustados aos tempos da pandemia, o que está acontecendo na maioria dos municípios 
brasileiros. Com isto, o setor se somou a outros no combate ao coronavírus, ao possibilitar a circulação das pessoas necessárias a denominada "economia de guerra", e caso contrário, milhões de pessoas teriam ficado desassistidas. Porém, essa primeira medida se desdobra em ações que precisam ser aperfeiçoadas ao longo da pandemia, tais como o ajuste das redes e a oferta de serviços de forma a assegurar e promover a redução da superlotação de veículos, criação de novos serviços envolvendo as prefeituras e empresas operadoras, como linhas de ônibus e micro-ônibus, que são destinados, principalmente, ao deslocamento de trabalhadores e pacientes para os equipamentos de saúde e assistência social (Tirachini et al., 2020).

Outrossim, a facilitação da integração nos terminais de ônibus com bicicletas, motos e serviços de aplicativos precisa ser melhor aperfeiçoada, bem como o auxílio na locomoção imediata de pessoas infectadas das comunidades para os centros de saúde e abrigos seguros. Ademais, as empresas do setor precisam proteger seus empregadores e buscar todos os meios para manter seus empregos, e, para isso, deve-se informar os profissionais das empresas sobre a pandemia e cuidados a serem tomados, garantir o fornecimento de equipamentos de proteção individual (EPIs) para motoristas, cobradores e demais trabalhadores do setor. Além disso, deve-se adotar processos de higienização dos veículos, paradas e terminais, de maneira constante (Wang et al., 2020).

Aliado a isso, cabe ao Estado e autoridades municipais de regiões metropolitanas a promoção da coordenação das ações durante a crise, o que pode envolver o ajuste de redes de serviço e promoção da integração entre diferentes modos de transporte, destinadas a facilitar o deslocamento intermunicipal, como também a garantia de pleno funcionamento do sistema de metrôs, trens regionais e outros sistemas veiculares geridos pelos Estados, aplicando a eles as mesmas exigências de cuidado dos profissionais envolvidos, de medidas de higiene de veículos e estações, bem como do monitoramento da lotação desses transportes (Klueber et al., 2020).

Sob essa perspectiva, é importante salientar a importância de se manter canais abertos entre municípios, empresas e fornecedores, para que a comunicação acerca do funcionamento do transporte público seja objetiva, coerente e transparente. E, finalmente, a participação durante o setor durante o cenário pandêmico enseja de ações voltadas à sociedade como um todo, tais como a disponibilização de veículos e motoristas para o transporte de insumos alimentares e de limpeza para as comunidades, assim como, em sentido inverso, contribuir para a distribuição de produtos realizados nas comunidades, como a distribuição de máscaras de proteção produzidas por comunidades de costureiras (Laverty et al., 2020).

\section{Considerações Finais}

A pandemia de COVID-19 provocou inúmeras mudanças na vida da população mundial. Na mobilidade urbana não foi diferente. Com o fechamento do comércio considerado não essencial, a suspensão de aulas e a instalação de regimes de teletrabalho, grande parte dos deslocamentos diários foram interrompidos e/ou modificados. Medidas como a instalação de políticas de subsídio do transporte público, de ampliação das ciclovias, de aplicação de ações de desestímulo do uso do transporte individual motorizado, de melhora na segurança pública e de circulação de pedestres e ciclistas etc., se mostraram urgentes durante a quarentena, não apenas para gerar condições mínimas para mobilidade ativa, mas também para melhorar a qualidade de vida da população como um todo.

\section{Referências}

Aloi, A., et al. (2020). Efeitos do bloqueio COVID-19 na mobilidade urbana: evidências empíricas da cidade de Santander (Espanha). Sustainability, 12 (4), 3870-3874.

Arellana, J., et al. (2020). Surto de COVID-19 na Colômbia: uma análise de seus impactos nos sistemas de transporte. J. Adv. Transp. 45 (8), 1-8.

Chinazzi, M., et al. (2020). O efeito das restrições de viagens na disseminação do novo surto de coronavírus (COVID-19) em 2019. Science, 368 (8), 395-400.

Hadjidemetriou, G. M., et al. (2021). O impacto das medidas governamentais e a tendência da mobilidade humana nas mortes relacionadas ao COVID-19 no Reino Unido. Transp. Res. Interdiscip. Perspect., 6 (8), 1-7. 
Research, Society and Development, v. 10, n. 16, e460101623917, 2021

(CC BY 4.0) | ISSN 2525-3409 | DOI: http://dx.doi.org/10.33448/rsd-v10i16.23917

Huang, J., et al. (2020). Understanding the Impact of the COVID-19 Pandemic on Transportation-related Behaviors with Human Mobility Data. Science, 370 (4), 343-350.

Kraemer, M. U. G., et al. (2020). O efeito da mobilidade humana e das medidas de controle na epidemia de COVID-19 na China. Science, 368 (8), $493-497$.

Kuebler, M. (2020). Como a pandemia está transformando a mobilidade urbana. DW. Lancet, 41 (6), 4-7.

Laverty, A. A., et al. (2020). COVID-19 apresenta oportunidades e ameaças ao transporte e à saúde. JR Soc. Med., 113 (4), 251-254.

Tirachini, A., et al. (2020). COVID-19 e transporte público: Avaliação atual, perspectivas e necessidades de pesquisa. J. Public Transp., 22 (3), 1-34.

Wang, X., et al. (2020). Análise de vulnerabilidade e resiliência da rede do setor de controle de tráfego aéreo na China. Sustainability, 12 (1), $37-49$.

Ximenes, D. S., et al. (2020). Emoções momentâneas: comportamentos e hábitos cotidianos pós-pandemia. Revista do Centro de Síntese USP Cidades Globais do IEA/USP, 1-13. 